\title{
Increased Plasma Matrix Metalloproteinase-9 Levels Contribute to Intracerebral Hemorrhage during Thrombolysis after Concomitant Stroke and Influenza Infection
}

\author{
Sajjad Muhammad ${ }^{a} d \quad$ Oliver Planz $^{b}$ Markus Schwaninger ${ }^{c, d}$ \\ a Department of Neurosurgery, University Hospital Bonn, Bonn, ${ }^{b}$ Department of \\ Immunology, University of Tübingen, Tübingen, 'Institute of Experimental and Clinical \\ Pharmacology and Toxicology, University of Lübeck, Lübeck, and d Department of \\ Pharmacology, University of Heidelberg, Heidelberg, Germany
}

Key Words

Stroke $\cdot$ Infection · Matrix metalloproteinase-9 $\cdot$ Thrombolysis $\cdot$ Intracerebral bleeding

\begin{abstract}
Background: Thrombolysis is the only approved therapy for acute stroke. However, life-threatening complications such as intracerebral hemorrhage (ICH) can develop after intravenous administration of tissue plasminogen activator (tPA). Both infection and thrombolysis during cerebral ischemia disrupt the blood-brain barrier (BBB). tPA can induce matrix metalloproteinase-9 (MMP-9), which is known to be involved in BBB disruption. However, it has still not been investigated whether preexisting influenza virus infection during thrombolysis after acute stroke affects systemic levels of MMP-9 and its inhibitor TIMP-1 and whether increased systemic MMP-9 levels affect ICH. This study aimed to investigate the influence of influenza virus infection on plasma levels of MMP-9 and TIMP-1 after thrombolysis in acute stroke, and to determine whether the infection correlates with intracerebral bleeding. Methods: C57BL/6 mice were infected by administering $1 \times 10^{5}$ plaque-forming units of human influenza (H1N1) virus intranasally. After 3 days of infection the middle cerebral artery was occluded for $45 \mathrm{~min}$ and then reperfused. Intravenous tPA $(10 \mathrm{mg} / \mathrm{kg})$ treatment was started $10 \mathrm{~min}$ after stroke onset. Twenty-four hours after stroke onset, mice were deeply anesthetized with ketamine, venous blood was drawn from the caval vein and centrifuged at 2,000 rpm, and the supernatant was collected and frozen at $-80^{\circ} \mathrm{C}$. Plasma levels of MMP-9 and TIMP-1 were quantified by using ELISA. Results: After stroke, plasma MMP-9 was significantly increased in mice with a concomitant influenza infection that were treated with tPA $(9.99 \pm 0.62 \mathrm{ng} / \mathrm{ml}, \mathrm{n}=7)$ as compared to noninfected control mice that were treated with tPA $(4.74 \pm 0.48 \mathrm{ng} / \mathrm{ml}, \mathrm{n}=8)$. More-
\end{abstract}

Dr. med. Sajjad Muhammad

Department of Neurosurgery, University Hospital Bonn

Sigmund-Freud-Strasse 25

DE-53127 Bonn (Germany)

E-Mail sajjad.muhammad@ukb.uni-bonn.de 
over, plasma levels of TIMP-1, an inhibitor of MMP-9, were also significantly increased in mice treated with tPA after concomitant infection and stroke $(42.17 \pm 7.02 \mathrm{ng} / \mathrm{ml}, \mathrm{n}=7)$ as compared to noninfected control mice that were treated with tPA after stroke $(20.22 \pm 2.12 \mathrm{ng} / \mathrm{ml}, \mathrm{n}=8)$. MMP-9 values significantly correlated with intracerebral hemoglobin levels in animals treated with tPA after stroke $(p=0.028, r=0.76, n=8)$ and after concomitant stroke and infection ( $p=0.039, r=0.78, n=7)$. Conclusion: Preexisting influenza A virus infection led to increased plasma MMP-9 and TIMP-1 levels in mice undergoing thrombolysis after induced stroke. MMP9 levels closely correlated with intracerebral bleeding after thrombolysis during concomitant infection and stroke. Thus, our data indicate that thrombolysis may be dangerous during influenza infection. MMP-9 inhibitors might be considered to reduce the side effects of thrombolysis during concomitant infection and stroke.

\section{Introduction}

Thrombolysis is the only approved therapy for ischemic stroke within a time window of $4.5 \mathrm{~h}$ [1]. However, only a minority of patients receive thrombolysis because of the risk of lifethreatening complications, especially intracerebral hemorrhage (ICH). Even after strict selection of patients for thrombolysis, ICH has been reported in up to $7.7 \%$ of patients within the first $36 \mathrm{~h}$ after onset of stroke [2], and despite improvements in thrombolytic therapy, ICH is still the major cause of death in the acute phase of stroke after intravenous thrombolysis [3]. Neuroprotective drugs have failed in clinical trials; therefore, improving thrombolytic therapy has become even more important. Activation of matrix metalloproteinase-9 (MMP-9) plays a key role in blood-brain barrier (BBB) disruption and the development of ICH after intravenous thrombolysis. Interestingly, MMP-9 inhibitors alleviate bleeding complications of thrombolysis in animal models of stroke [4, 5]. ICH after stroke is, however, multifactorial [6]. We have recently shown that preexisting systemic influenza infection can exacerbate intracerebral bleeding after thrombolysis [7]. However, it is not known whether a concomitant infection affects systemic MMP-9 levels and whether plasma MMP-9 levels correlate with intracerebral bleeding after intravenous thrombolysis.

Systemic inflammation due to peripheral infection increases systemic concentrations of proinflammatory cytokines, including CCL5 and IL-1 $\beta$. Moreover, systemic inflammation can activate microvascular MMPs and disrupt the BBB, which then promotes infiltration of neutrophils [7-9]. Neutrophils release MMP-9, which degrades collagen IV in the basement membrane and increases vascular permeability. We have recently shown that, under these conditions, thrombolysis induced significantly more intracerebral bleeds [7]. Blood biomarkers could be important for identifying patients at increased risk of intracerebral bleeding. Preclinical studies have shown that MMP-9 expression is increased in the brain during thrombolysis; however, the significance of plasma MMP-9 levels during thrombolysis and concomitant infection has not been investigated.

To analyze the effect of influenza infection on plasma levels of MMP-9 and its inhibitor TIMP-1 after thrombolysis, we infected mice with influenza A virus and induced stroke by middle cerebral artery occlusion (MCAO). Plasma levels of MMP-9 and its tissue inhibitor TIMP-1 were determined. Influenza infection increased plasma MMP-9 levels in mice treated with tissue plasminogen activator (tPA) after stroke. Mice treated with tPA after concomitant infection and stroke showed significantly increased bleeding in the brain [7]. Interestingly, MMP-9 values closely correlated with intracerebral bleeding in animals treated with tPA after concomitant stroke and infection. Our data provide clinically important information for thrombolytic therapy of acute stroke in conditions associated with peripheral inflammation/infection. 


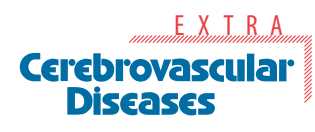

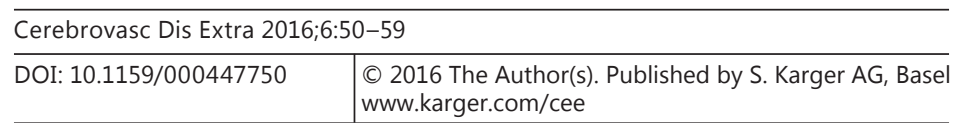

Muhammad et al.: Increased Plasma MMP-9 Levels Contribute to Intracerebral Hemorrhage during Thrombolysis after Concomitant Stroke and Influenza Infection

\section{Methods}

All animal experiments were approved by the local animal welfare committee. The animals were randomly assigned to four groups with 10 animals in each group. The investigator was blinded to treatment. Animals with confirmed clinical symptoms of influenza were included in the study. Dead animals or animals that did not develop clinical symptoms of influenza were excluded. We had a mortality rate of around $20 \%$ and could analyze $7-8$ animals per group. The data for infarct volumes and blood volume/hemorrhagic scores for correlation with MMP-9 levels were taken from a previously reported study [7].

\section{Influenza Virus and Infection}

Human influenza virus A/Puerto Rico/8/34 (H1N1; PR8) was propagated as described previously [10]. Male C57BL/6 mice aged 3-4 months were anesthetized by i.p. injection of xylazine $(50 \mu \mathrm{l} / 10 \mathrm{~g}$ body weight, $0.1 \%)$ and ketamine ( $50 \mu \mathrm{l} / 10 \mathrm{~g}$ body weight, $0.5 \%)$, unless indicated otherwise. We administered the H1N1 virus $\left(1 \times 10^{5}\right.$ plaque-forming units $)$ or the control inoculum (PBS) in a final volume of $50 \mu \mathrm{l}$ (25 $\mu \mathrm{l}$ in each nasal opening). Successful infection was confirmed by virus titration of the lungs $(n=5)$ [7].

\section{MCAO}

In all experiments, the animals were randomized and the investigators were blinded to the treatment. For transient MCAO (tMCAO), male mice were anesthetized at an age of 3-4 months by i.p. injection of xylazine $(50 \mu \mathrm{l} / 10 \mathrm{~g}$ body weight, $0.1 \%)$ and ketamine $(50 \mu \mathrm{l} / 10 \mathrm{~g}$ body weight, $0.5 \%$ ). A median neck incision was made and the left common carotid artery was exposed. A silicon rubber-coated 7-0 monofilament $20 \mathrm{~mm}$ in length (Doccol Corp., Cat. No. 7019PK5Re) was inserted into the common carotid artery on the left side. The filament was advanced into the internal carotid artery about $11 \mathrm{~mm}$ from the bifurcation until the coated part of the filament was no longer visible. The filament was reversibly fixed for the duration of ischemia. After $45 \mathrm{~min}$ of occlusion, the filament was removed to reestablish perfusion. During the time of occlusion, the femoral vein was exposed and cannulated with a 20-cm-long polyethylene catheter (PE-10). Human tPA $(10 \mathrm{mg} / \mathrm{kg}$ at a volume of $300 \mu \mathrm{l}$; Boehringer Ingelheim, Germany) was administered starting $30 \mathrm{~min}$ after MCAO with a bolus (1 mg/kg). The remaining dose $(9 \mathrm{mg} / \mathrm{kg}$ ) was infused over $20 \mathrm{~min}$ with a pump (PHD2000 Programmable; Harvard Apparatus). At $24 \mathrm{~h}$ after tMCAO the animals were reanesthetized and perfused intracardially with $4 \%$ PFA.

\section{Hemoglobin Assay}

To measure the hemoglobin content in the brain, we performed a spectrophotometric assay using Drabkin's solution. Briefly, the animals were perfused transcardially $24 \mathrm{~h}$ after tMCAO with PFA (4\%, $20 \mathrm{ml}$ with a perfusion speed of $1.5 \mathrm{ml} / \mathrm{min})$. To prepare standards, fresh blood was injected into the cortex immediately before perfusion. The brains were homogenized by sonication in $1 \mathrm{ml}$ PBS per hemisphere. After centrifugation, $120 \mu \mathrm{l}$ of Drabkin's solution containing Drabkin's reagent (Sigma, Cat. No. D5941) and Brij 35 solution $(0.015 \%$, Sigma, Cat. No. B 4184$)$ was added to $30 \mu \mathrm{l}$ of supernatant and the absorption was measured at $540 \mathrm{~mm}$ (Spectra MAX 250, Molecular Devices Corp.) [7].

\section{Measurement of MMP-9 and TIMP-1}

To determine MMP-9 and TIMP-1 plasma levels, blood samples were obtained $24 \mathrm{~h}$ after tMCAO. ELISA was performed according to the manufacturer's instructions (MMP-9, USCN Life Science Inc., Wuhan, Cat. No. E0553Mu; TIMP-1, RayBiotech, Inc., Cat. No. ELM-TIMP1001). To quantify TIMP-1 in the plasma, the samples were diluted 1:50. 
Fig. 1. Plasma MMP-9 (a) and TIMP-1 (b) levels increased significantly after $24 \mathrm{~h}$ of ischemia and thrombolysis in the presence of influenza $A$ virus infection (one-way ANOVA, $\mathrm{n}=7-8, \mathrm{p}<$ 0.05 ).

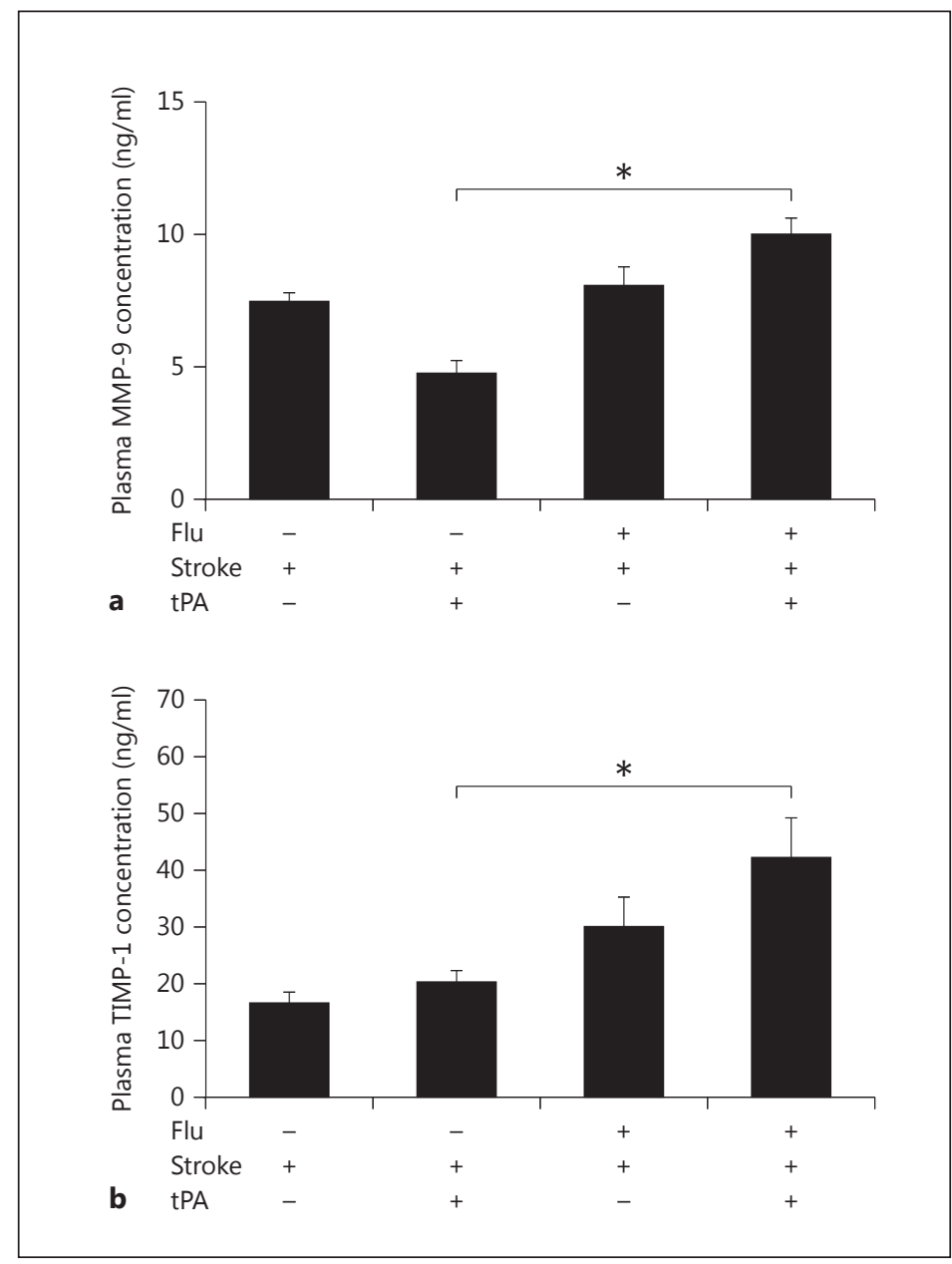

\section{Statistics}

One-way ANOVA was used to compare more than two groups, followed by the NewmanKeuls multiple comparison test. We calculated Pearson's correlation coefficient. Data are expressed as means \pm SEM.

\section{Results}

Plasma MMP-9 and Its Tissue Inhibitor TIMP-1 Were Elevated during Thrombolytic

Therapy after Concomitant Infection and Stroke

MMP-9 is known to degrade the extracellular matrix and may contribute to BBB disruption. Hence, we analyzed plasma concentrations of MMP-9 and its inhibitor TIMP-1. To evaluate whether influenza virus infection influences plasma MMP-9 levels after thrombolytic treatment of stroke and concomitant infection, we employed a stroke model (tMCAO) that had previously been used to investigate the complications of tPA treatment $[7,11]$. We administered tPA 30 min after occlusion. MMP-9 levels were significantly elevated in a group of animals that were treated with tPA for stroke in the presence of an influenza A infection (9.99 $\pm 0.62 \mathrm{ng} / \mathrm{ml}, \mathrm{n}=7$ ) as compared to control mice $(4.74 \pm 0.48 \mathrm{ng} / \mathrm{ml}, \mathrm{n}=8$ ) without any infection (fig. 1a). Interestingly, intracerebral blood volume was significantly increased 

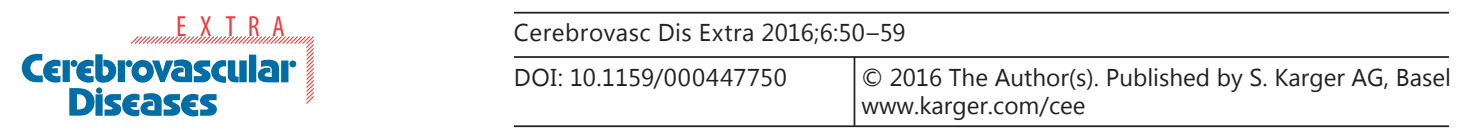

Muhammad et al.: Increased Plasma MMP-9 Levels Contribute to Intracerebral

Hemorrhage during Thrombolysis after Concomitant Stroke and Influenza Infection

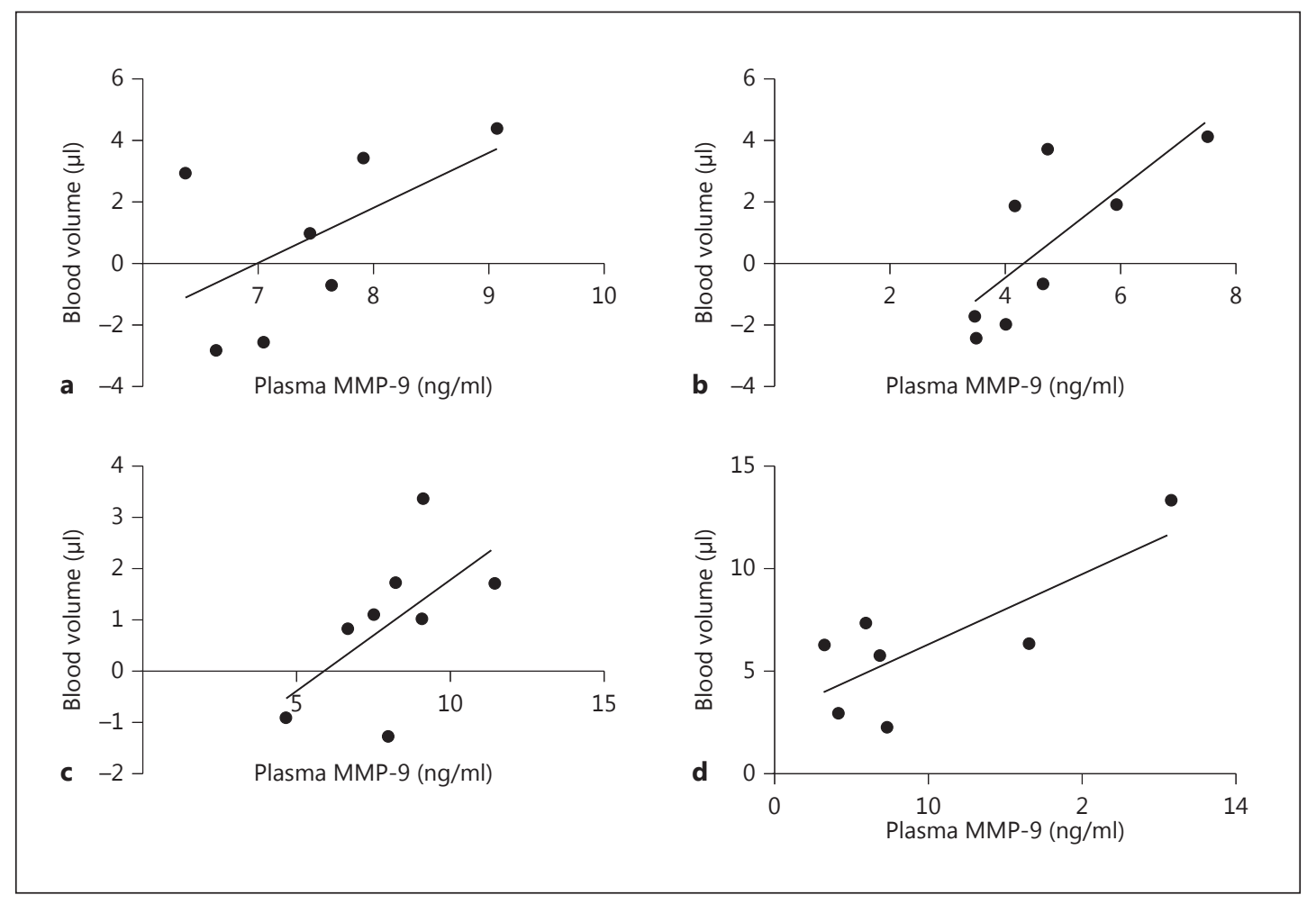

Fig. 2. Plasma MMP-9 levels showed correlation with intracranial blood volume in mice treated with i.v. thrombolysis after stroke without infection (Pearson correlation, $n=8, r=0.76, p=0.027$ ) and in the presence of influenza A virus infection (Pearson correlation, $n=7, r=0.78, p=0.039$ ). Plasma MMP-9 levels showed no statistically significant correlation in noninfected animals $(n=7, r=0.55, p=0.19)$ and infected animals $(\mathrm{n}=8, \mathrm{r}=0.57, \mathrm{p}=0.13)$ without i.v. thrombolysis. a Stroke. b Stroke plus thrombolysis. $\mathbf{c}$ Stroke plus influenza infection. d Stroke plus thrombolysis plus influenza infection.

during thrombolysis after concomitant infection and stroke (to $5.98 \pm 1.24 \mu \mathrm{l}$ in comparison to $0.63 \pm 0.93 \mu$ in noninfected mice) as reported previously [7]. Similarly, TIMP-1 plasma levels were significantly higher in animals that were treated with tPA for stroke in the presence of an influenza A infection $(42.17 \pm 7.02 \mathrm{ng} / \mathrm{ml}, \mathrm{n}=7)$ than in control mice $(20.22 \pm$ $2.12 \mathrm{ng} / \mathrm{ml}, \mathrm{n}=8$ ) without any infection (fig. 1b). These data show that MMP-9 and its inhibitor TIMP-1 were upregulated after concomitant infection, stroke, and thrombolysis.

\section{Plasma MMP-9 Levels Significantly Correlated with Thrombolysis-Mediated ICH during}

Concomitant Stroke and Influenza Infection

Thrombolysis is known to increase systemic MMP-9 levels, and MMP-9 is a key mediator of BBB disruption, which leads to ICH $[6,12,13]$. Our data demonstrate that there was no significant increase in plasma MMP-9 levels after influenza infection alone (fig. 1a). However, thrombolysis in the presence of influenza infection dramatically increased systemic MMP-9 levels (fig. 1a) and thrombolysis-mediated ICH [7], showing that a combination of infection and thrombolysis during acute stoke may be dangerous. Interestingly, MMP-9 plasma concentrations significantly correlated with the intracerebral blood volume (fig. 2). Further analysis showed no correlation between plasma MMP-9 and infarct volume (fig. 3) or between intracerebral blood volume and infarct volume (fig. 4). The hemorrhagic score on brain sections was quantified [7] in infected and noninfected mice after stroke with and without throm- 


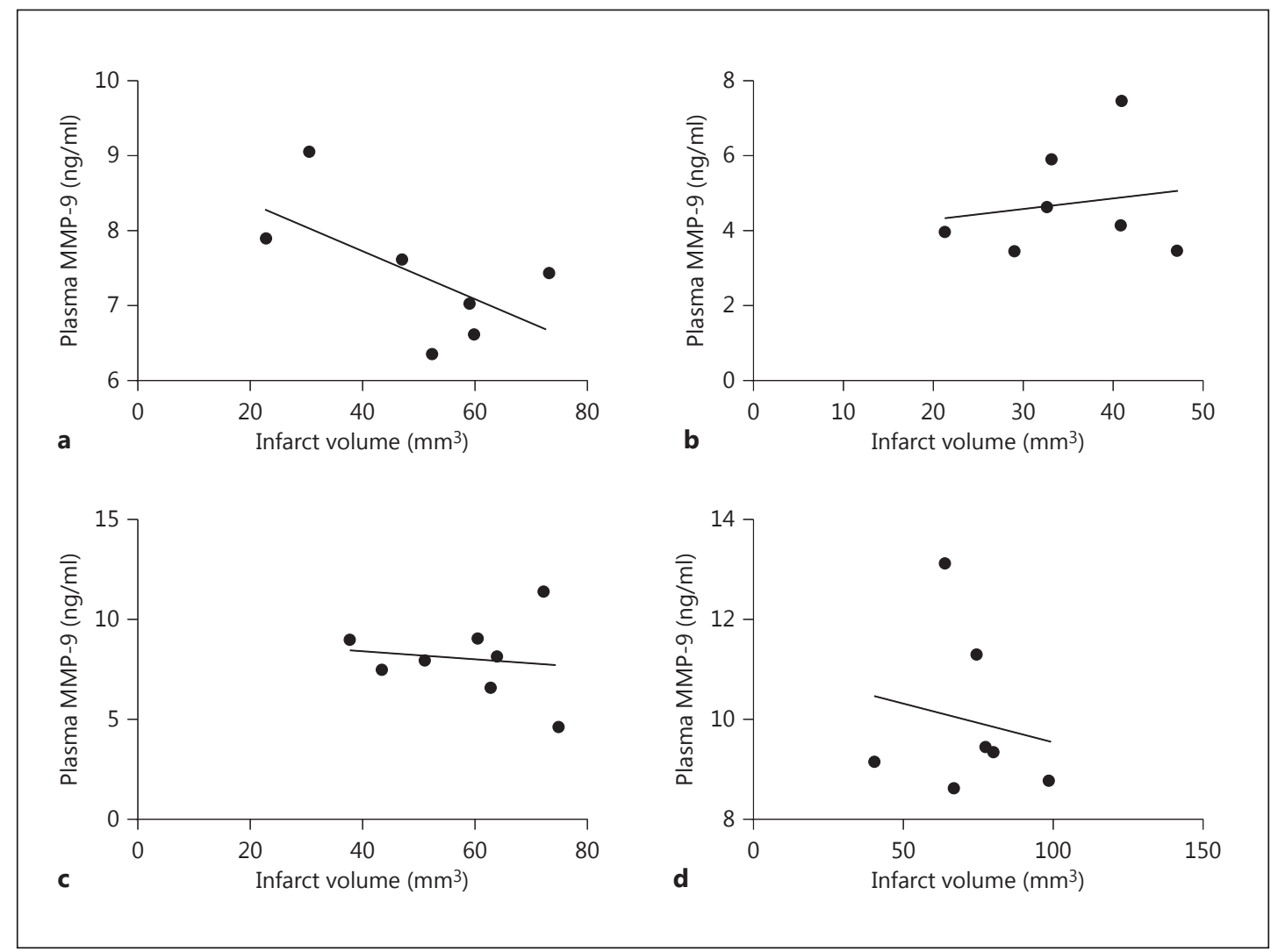

Fig. 3. Plasma MMP-9 levels showed no statistically significant correlation with infarct volume in mice after stroke in the presence or absence of influenza A virus infection and i.v. thrombolysis (Pearson correlation, $\mathrm{n}=7-8)$. a Stroke. $\mathbf{b}$ Stroke plus thrombolysis. c Stroke plus influenza infection. $\mathbf{d}$ Stroke plus thrombolysis plus influenza infection.

bolysis. It was significantly increased after infection and stroke during thrombolysis [7]. However, hemorrhagic scores on sections did not correlate with plasma MMP-9 (fig. 5). The reason for this lack of correlation might lie in the limited sensitivity and linearity of scoring hemorrhages on histological sections. Our data suggest that plasma MMP-9 may be responsible for intracerebral bleeding during thrombolysis in subjects with an influenza infection.

\section{Discussion}

In the clinical setting, MMP-9 plasma levels increase gradually after tPA treatment of

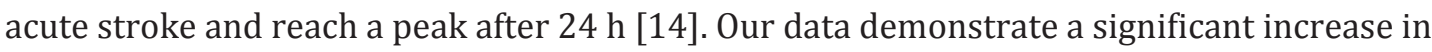
MMP-9 levels $24 \mathrm{~h}$ after cerebral ischemia and tPA treatment in influenza-infected mice as compared to control mice treated with tPA after acute stroke without any infection (fig. 1a). MMP-9, a protease that can be induced by thrombin, can be neurotoxic and increase the risk of ICH. Pretreatment with MMP-9 in an experimental setting has been shown to increase hemorrhagic transformation after ischemia [15]. Systemic MMP-9 and its tissue inhibitor TIMP-1 are known to play an important role during BBB disruption. In human patients, influenza virus infection led to increased systemic MMP-9 levels [16,17]. A concomitant influenza virus infection altered systemic cytokines, increased infiltration of MMP-9-positive 

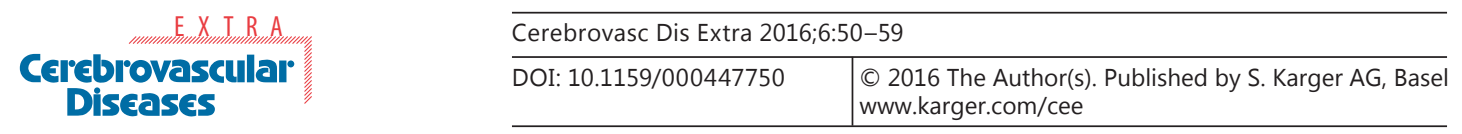

Muhammad et al.: Increased Plasma MMP-9 Levels Contribute to Intracerebral

Hemorrhage during Thrombolysis after Concomitant Stroke and Influenza Infection

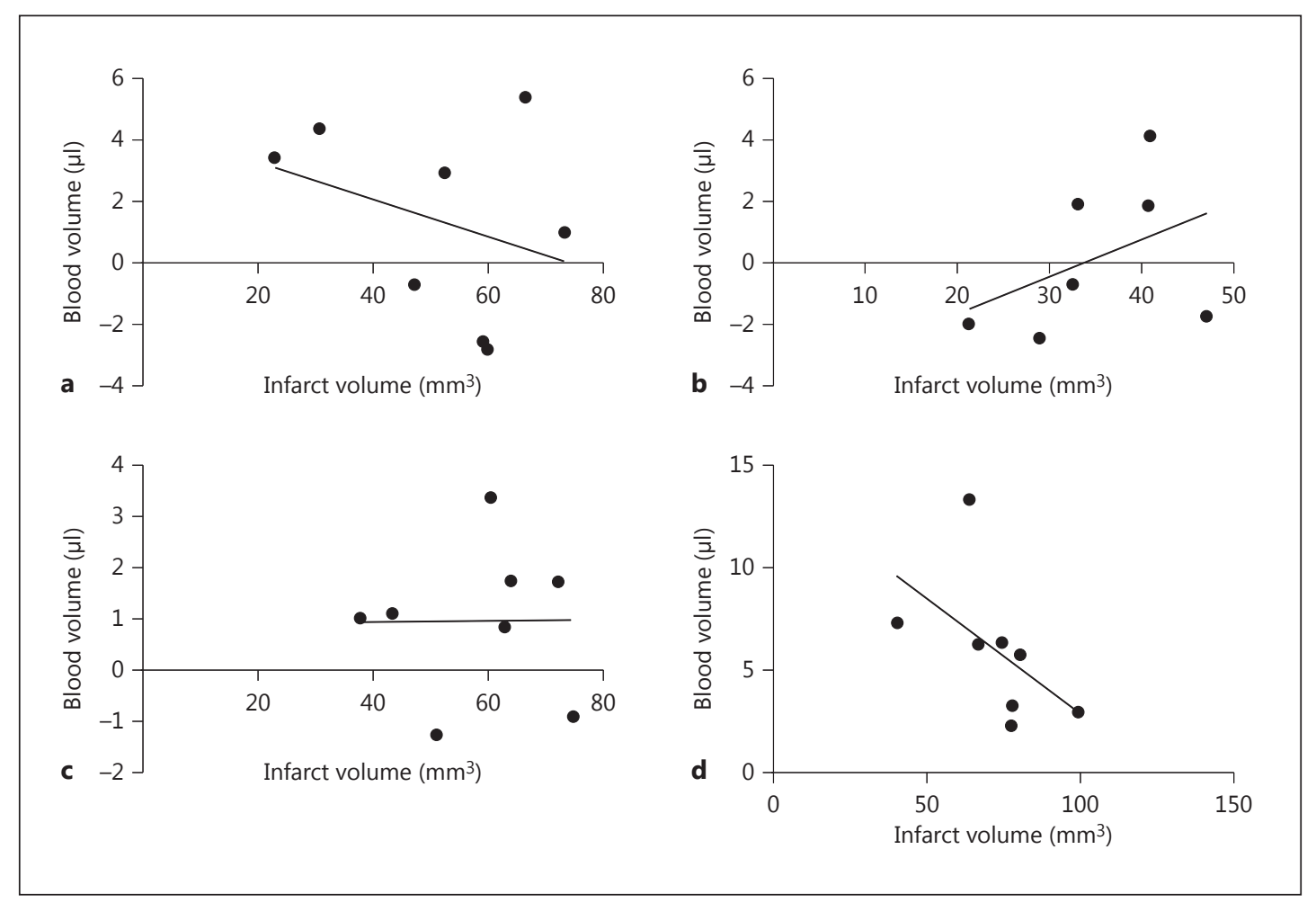

Fig. 4. Intracerebral blood volume showed no statistically significant correlation with infarct volume in mice after stroke in the presence or absence of influenza A virus infection and i.v. thrombolysis (Pearson correlation, $\mathrm{n}=7-8$ ) a Stroke. b Stroke plus thrombolysis. c Stroke plus influenza infection. d Stroke plus thrombolysis plus influenza infection.

leukocytes in the brain and aggravated the breakdown of collagen IV in the basement membrane that ultimately disrupts the BBB in cerebral ischemia [7]. Interestingly, influenza infection did not change physiological parameters such as arterial blood pressure, blood flow (as measured by laser Doppler), blood gases, base excess, blood glucose, etc. in this model setting [7]. The mechanism underlying ICH after cerebral ischemia is, however, a multifactorial phenomenon, leading to additional brain injury and ultimately to a poor clinical outcome $[6,12]$. Lipoprotein receptor-mediated induction of MMP-9 by tPA has been shown to be a key mechanism for increasing BBB permeability and for the increased risk of ICH after thrombolysis $[18,19]$. Infiltration of MMP-9-positive neutrophils after ischemia and neutrophil degranulation represents a critical step in exacerbating the vascular injury [12]. We have demonstrated that systemic inflammation increases neutrophil infiltration and ICH [7]. Interestingly, the presence of influenza virus infection potentiated the systemic levels of MMP-9 and its tissue inhibitor TIMP-1 after thrombolysis (fig. 1a, b). Our results and data from the literature suggest that it is relevant to identify the factors that can potentiate MMP-9 release during thrombolysis to predict the post-thrombolytic risk of hemorrhage. Serum uric acid levels can predict ICH and clinical outcome after thrombolysis [20]. Mechanistically, a decrease in serum uric acid levels has been shown to increase neutrophil infiltration and MMP-9 release. A pilot study has shown that additional treatment with uric acid reduced thrombolysis-mediated ICH [21]. Experimental studies suggest that additional treatment with MMP inhibitors such as BB-94, GM 6001 and p-aminobenzoyl-gly-pro-D-leu-D-ala hydroxamate represents an effective option to reduce the side effects of thrombolysis [4, 

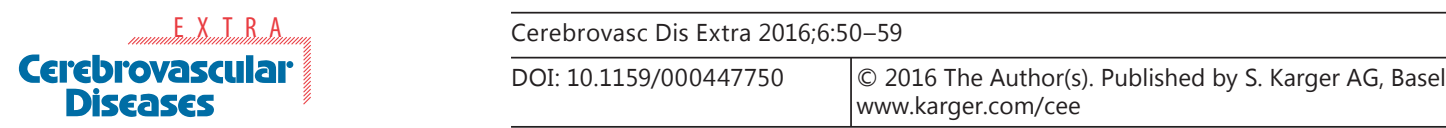

Muhammad et al.: Increased Plasma MMP-9 Levels Contribute to Intracerebral Hemorrhage during Thrombolysis after Concomitant Stroke and Influenza Infection

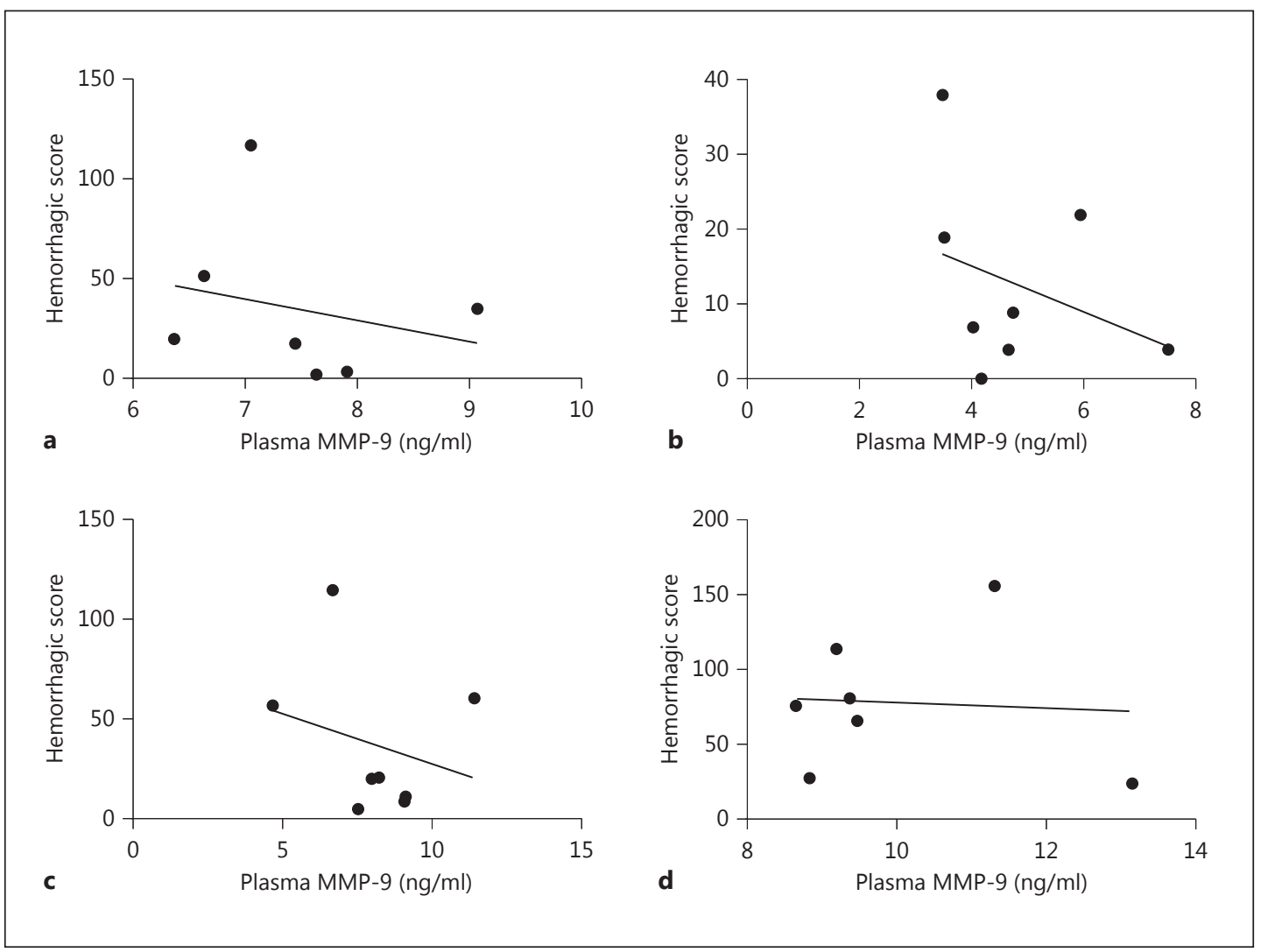

Fig. 5. The hemorrhagic score on brain sections showed no statistically significant correlation with plasma MMP-9 in mice after stroke in the presence or absence of influenza A virus infection and i.v. thrombolysis (Pearson correlation, $\mathrm{n}=7-8$ ). a Stroke. b Stroke plus thrombolysis. c Stroke plus influenza infection. d Stroke plus thrombolysis plus influenza infection.

22-24]. Interestingly, 17 $\beta$-estradiol and doxycycline improved microvascular stabilization and decreased thrombolysis-mediated ICH by inhibiting MMPs $[25,26]$. The free radical scavenger edaravone reduced MMP-9-related ICH after thrombolysis [27]. Our data, in line with the literature, show that post-thrombolytic MMP-9 plasma levels during concomitant infection and stroke showed a significant correlation with ICH (fig. 2d). However, we analyzed the total plasma MMP-9 and not the active MMP-9, which is certainly a limitation of our study. As thrombolysis is the only approved therapeutic option and under certain conditions (such as influenza infection) life-threatening side effects can develop, MMP-9 inhibitors might be considered to increase the safety of thrombolysis in such cases.

\section{Conclusion}

Preexisting influenza A virus infection increased plasma MMP-9 levels in mice undergoing thrombolysis after stroke. Increased plasma MMP-9 levels correlated with intracerebral bleeding after thrombolysis during concomitant infection and stroke. Thrombolysis after stroke in the presence of influenza infection may be dangerous. Combined therapeutic approaches with MMP-9 inhibitors and tPA could be developed and considered so as to reduce the side effects of thrombolysis. 
Muhammad et al.: Increased Plasma MMP-9 Levels Contribute to Intracerebral

Hemorrhage during Thrombolysis after Concomitant Stroke and Influenza Infection

\section{Acknowledgments}

The research leading to these results received funding from the European Union's Seventh Framework Program (FP7/2007-2013) under grant agreements 201024 and 202213 (European Stroke Network), from the Federal Government of Germany under the Influenza Research Program 'FSI', from the Postdoc program of the Medical Faculty, University of Heidelberg, and from the BMBF Zoonose program 'FluResearchNet' (01KI07141).

\section{Disclosure Statement}

The authors have no conflicts of interest to declare.

\section{References}

1 Lees KR, Bluhmki E, von Kummer R, Brott TG, Toni D, Grotta JC, Albers GW, Kaste M, Marler JR, Hamilton SA, Tilley BC, Davis SM, Donnan GA, Hacke W, Allen K, Mau J, Meier D, del Zoppo G, De Silva DA, Butcher KS, Parsons MW, Barber PA, Levi C, Bladin C, Byrnes G: Time to treatment with intravenous alteplase and outcome in stroke: an updated pooled analysis of ECASS, ATLANTIS, NINDS, and EPITHET trials. Lancet 2010;375:1695-1703.

-2 Wardlaw JM, Murray V, Berge E, del Zoppo G, Sandercock P, Lindley RL, Cohen G: Recombinant tissue plasminogen activator for acute ischaemic stroke: an updated systematic review and meta-analysis. Lancet 2012; 379:2364-2372.

-3 Sandercock P, Wardlaw JM, Lindley RI, Dennis M, Cohen G, Murray G, Innes K, Venables G, Czlonkowska A, Kobayashi A, Ricci S, Murray V, Berge E, Slot KB, Hankey GJ, Correia M, Peeters A, Matz K, Lyrer P, Gubitz G, Phillips SJ, Arauz A: The benefits and harms of intravenous thrombolysis with recombinant tissue plasminogen activator within $6 \mathrm{~h}$ of acute ischaemic stroke (the third international stroke trial [IST-3]): a randomised controlled trial. Lancet 2012;379:2352-2363.

4 Copin JC, Merlani P, Sugawara T, Chan PH, Gasche Y: Delayed matrix metalloproteinase inhibition reduces intracerebral hemorrhage after embolic stroke in rats. Exp Neurol 2008;213:196-201.

5 Murata Y, Rosell A, Scannevin RH, Rhodes KJ, Wang X, Lo EH: Extension of the thrombolytic time window with minocycline in experimental stroke. Stroke 2008;39:3372-3377.

6 Rosell A, Foerch C, Murata Y, Lo EH: Mechanisms and markers for hemorrhagic transformation after stroke. Acta Neurochir Suppl 2008;105:173-178.

7 Muhammad S, Haasbach E, Kotchourko M, Strigli A, Krenz A, Ridder DA, Vogel AB, Marti HH, Al-Abed Y, Planz O, Schwaninger M: Influenza virus infection aggravates stroke outcome. Stroke 2011;42:783-791.

8 Denes A, Humphreys N, Lane TE, Grencis R, Rothwell N: Chronic systemic infection exacerbates ischemic brain damage via a CCL5 (regulated on activation, normal T-cell expressed and secreted)-mediated proinflammatory response in mice. J Neurosci 2010;30:10086-10095.

9 McColl BW, Rothwell NJ, Allan SM: Systemic inflammation alters the kinetics of cerebrovascular tight junction disruption after experimental stroke in mice. J Neurosci 2008;28:9451-9462.

10 Ehrhardt C, Wolff T, Pleschka S, Planz O, Beermann W, Bode JG, Schmolke M, Ludwig S: Influenza A virus NS1 protein activates the PI3K/Akt pathway to mediate antiapoptotic signaling responses. J Virol 2007;81:30583067.

11 Cheng T, Petraglia AL, Li Z, Thiyagarajan M, Zhong Z, Wu Z, Liu D, Maggirwar SB, Deane R, Fernandez JA, LaRue B, Griffin JH, Chopp M, Zlokovic BV: Activated protein C inhibits tissue plasminogen activator-induced brain hemorrhage. Nat Med 2006;12:1278-1285.

-12 Rosell A, Cuadrado E, Ortega-Aznar A, Hernandez-Guillamon M, Lo EH, Montaner J: MMP-9-positive neutrophil infiltration is associated to blood-brain barrier breakdown and basal lamina type IV collagen degradation during hemorrhagic transformation after human ischemic stroke. Stroke 2008;39:1121-1126.

13 Sumii T, Lo EH: Involvement of matrix metalloproteinase in thrombolysis-associated hemorrhagic transformation after embolic focal ischemia in rats. Stroke 2002;33:831-836.

14 Zhao HD, Zhang YD: The effects of previous statin treatment on plasma matrix metalloproteinase-9 level in Chinese stroke patients undergoing thrombolysis. J Stroke Cerebrovasc Dis 2014;23:2788-2793.

15 Montaner J, Molina CA, Monasterio J, Abilleira S, Arenillas JF, Ribo M, Quintana M, Alvarez-Sabin J: Matrix metalloproteinase-9 pretreatment level predicts intracranial hemorrhagic complications after thrombolysis in human stroke. Circulation 2003;107:598-603.

16 Ichiyama T, Morishima T, Kajimoto M, Matsushige T, Matsubara T, Furukawa S: Matrix metalloproteinase-9 and tissue inhibitors of metalloproteinases 1 in influenza-associated encephalopathy. Pediatr Infect Dis J 2007;26:542-544. 
17 Sun G, Ota C, Kitaoka S, Chiba Y, Takayanagi M, Kitamura T, Yamamoto K, Fujie H, Mikami H, Uematsu M, HinoFukuyo N, Munakata M, Kure S, Haginoya K: Elevated serum levels of neutrophil elastase in patients with influenza virus-associated encephalopathy. J Neurol Sci 2015;349:190-195.

-18 Wang X, Lee SR, Arai K, Tsuji K, Rebeck GW, Lo EH: Lipoprotein receptor-mediated induction of matrix metalloproteinase by tissue plasminogen activator. Nat Med 2003;9:1313-1317.

19 Yepes M, Sandkvist M, Moore EG, Bugge TH, Strickland DK, Lawrence DA: Tissue-type plasminogen activator induces opening of the blood-brain barrier via the LDL receptor-related protein. J Clin Invest 2003;112:15331540.

20 Amaro S, Urra X, Gomez-Choco M, Obach V, Cervera A, Vargas M, Torres F, Rios J, Planas AM, Chamorro A: Uric acid levels are relevant in patients with stroke treated with thrombolysis. Stroke 2010;42(suppl 1):S28-S32.

21 Amaro S, Soy D, Obach V, Cervera A, Planas AM, Chamorro A: A pilot study of dual treatment with recombinant tissue plasminogen activator and uric acid in acute ischemic stroke. Stroke 2007;38:2173-2175.

-22 Lapchak PA, Chapman DF, Zivin JA: Metalloproteinase inhibition reduces thrombolytic (tissue plasminogen activator)-induced hemorrhage after thromboembolic stroke. Stroke 2000;31:3034-3040.

-23 Mishiro K, Ishiguro M, Suzuki Y, Tsuruma K, Shimazawa M, Hara H: A broad-spectrum matrix metalloproteinase inhibitor prevents hemorrhagic complications induced by tissue plasminogen activator in mice. Neuroscience 2012;205:39-48.

24 Pfefferkorn T, Rosenberg GA: Closure of the blood-brain barrier by matrix metalloproteinase inhibition reduces rtPA-mediated mortality in cerebral ischemia with delayed reperfusion. Stroke 2003;34:2025-2030.

25 Burggraf D, Trinkl A, Dichgans M, Hamann GF: Doxycycline inhibits MMPs via modulation of plasminogen activators in focal cerebral ischemia. Neurobiol Dis 2007;25:506-513.

-26 Liu R, Liu Q, He S, Simpkins JW, Yang SH: Combination therapy of 17beta-estradiol and recombinant tissue plasminogen activator for experimental ischemic stroke. J Pharmacol Exp Ther 2010;332:1006-1012.

-27 Yagi K, Kitazato KT, Uno M, Tada Y, Kinouchi T, Shimada K, Nagahiro S: Edaravone, a free radical scavenger, inhibits MMP-9-related brain hemorrhage in rats treated with tissue plasminogen activator. Stroke 2009;40: 626-631. 